\title{
Object Oriented Protoconcepts and Logics for Double and Pure Double Boolean Algebras
}

\author{
Prosenjit Howlader and Mohua Banerjee ${ }^{(\bowtie)}$ \\ Department of Mathematics and Statistics, Indian Institute of Technology, \\ Kanpur 208016, India \\ \{prosen,mohua\}@iitk.ac.in
}

\begin{abstract}
The notion of a protoconcept in the framework of Yao's object oriented concepts is proposed. Approximations by object oriented concepts are defined and these 'object oriented protoconcepts' are characterized using them. It is shown that the object oriented protoconcepts form a double Boolean algebra, and any double Boolean algebra is quasiembedded in an algebra of protoconcepts. A logic DBL for the class of double Boolean algebras is proposed along with an extension PDBL for the class of pure double Boolean algebras. Utilizing the representation result for (pure) double Boolean algebras, it is established that DBL (PDBL) is sound and complete with respect to a semantics based on object oriented protoconcepts (semiconcepts).
\end{abstract}

\section{Introduction}

Formal concept analysis (FCA) [5] has seen wide applications since its inception. In order to study a conceptual knowledge system [10], Wille introduced the negation of a concept, leading to the notions of semiconcepts and protoconcepts. Algebraic studies of these notions led to the definition of double Boolean algebras and pure double Boolean algebras [11]. These structures have been investigated by many authors $[1,7-9]$.

Over the years, there has been a lot of work on different kinds of intersections of the theories of rough sets and FCA. Two seminal works in this regard are by Düntsch and Gediga, who introduced property oriented concepts [4], and by Yao, who proposed object oriented concepts [14]. Algebraic studies of these concepts have been conducted by many $[6,12,15]$. Our interest lies in introducing negation in the study of these concepts, in the line of the study by Wille as mentioned above. In [6], object oriented semiconcepts were introduced. In this work, we define object oriented protoconcepts (Sect.4), and characterize them through a notion of approximation by object oriented concepts. The algebraic structure formed by object oriented protoconcepts is studied giving a representation result

This work is supported by the Council of Scientific and Industrial Research (CSIR) India - Research Grant No. 09/092(0950)/2016-EMR-I.

(C) Springer Nature Switzerland AG 2020

R. Bello et al. (Eds.): IJCRS 2020, LNAI 12179, pp. 308-323, 2020.

https://doi.org/10.1007/978-3-030-52705-1_23 
for double Boolean algebras. In Sect.5, we propose a sequent calculus DBL, which is shown to be sound and complete with respect to the class of double Boolean algebras. DBL is extended to PDBL to give a logic for the class of pure double Boolean algebras. Utilizing the representation results for the algebras, it is next shown (Sect.6) that these logics are also sound and complete for semantics based on object oriented protoconcepts and semiconcepts respectively.

Preliminaries required for the work are presented in the next section. Section 3 revisits the notions of concepts and semiconcepts related to rough set theory, illustrated through a running example. Section 7 concludes the paper.

\section{FCA and Double Boolean Algebras}

Definition $1[5]$. A context is a triple $\mathbb{K}:=(G, M, R)$, where $G$ is the set of objects, $M$ is the set of properties, and $R \subseteq G \times M$.

For a context $\mathbb{K}:=(G, M, R)$, its complement is the context $\mathbb{K}^{c}:=$ $(G, M,-R)$ where $-R:=(G \times M) \backslash R$.

For any $A \subseteq G, B \subseteq M$, the following sets are defined:

$A^{\prime}:=\{m \in M: \forall g \in G(g \in A \Longrightarrow g R m)\}$, and

$B^{\prime}:=\{g \in G: \forall m \in M(m \in B \Longrightarrow g R m)\}$.

Then $(A, B)$ is a concept of $\mathbb{K}$, provided $A^{\prime}=B$ and $B^{\prime}=A$.

An order relation $\leq$ is defined on the set of all concepts as follows. For concepts $\left(A_{1}, B_{1}\right)$ and $\left(A_{2}, B_{2}\right),\left(A_{1}, B_{1}\right) \leq\left(A_{2}, B_{2}\right)$ if and only if $A_{1} \subseteq A_{2}$ (equivalently $B_{2} \subseteq B_{1}$ ).

Notation 1. We denote the class of all contexts by $\mathcal{K}$.

For a relation $R \subseteq G \times M, R^{-1}$ is the converse of $R$, that is $R^{-1} \subseteq M \times G$ and $y R^{-1} x$ if and only if $x R y$. For any $x \in G, y \in M, R(x):=\{y \in M: x R y\}$, and $R^{-1}(y):=\{x \in G: x R y\}$.

The complement of a subset $X$ of $G$ (or $M$ ) will be denoted simply by $X^{c}$.

The set of all concepts of the context $\mathbb{K}$ is denoted by $\mathcal{B}(\mathbb{K})$. For a concept $(A, B), A:=\operatorname{ext}((A, B))$ is its extent, while $B:=\operatorname{int}((A, B))$ is its intent.

Attempting to introduce the negation of a formal concept, it was noticed that there is a problem of closure if set-complement is used to define it. So the notion of concept was generalized to that of a semiconcept, and also to a protoconcept [11].

Definition 2. Let $\mathbb{K}:=(G, M, R)$ be a context and $A \subseteq G, B \subseteq M$. The pair $(A, B)$ is a semiconcept of $\mathbb{K}$ if and only if $A^{\prime}=B$ or $B^{\prime}=A$, while it is a protoconcept of $\mathbb{K}$ if and only if $A^{\prime \prime}=B^{\prime}$ (equivalently $A^{\prime}=B^{\prime \prime}$ ).

Notation 2. The set of all semiconcepts is denoted by $\mathfrak{H}(\mathbb{K})$ and the set of all protoconcepts is denoted by $\mathfrak{P}(\mathbb{K})$.

Observation 1. For a context $\mathbb{K}:=(G, M, R), \mathfrak{H}(\mathbb{K}) \subseteq \mathfrak{P}(\mathbb{K})$. 
The following operations are defined in $\mathfrak{P}(\mathbb{K})$. Let $\left(A_{1}, B_{1}\right),\left(A_{2}, B_{2}\right),(A, B)$ be any protoconcepts.

$$
\begin{aligned}
& \left(A_{1}, B_{1}\right) \sqcap\left(A_{2}, B_{2}\right):=\left(A_{1} \cap A_{2},\left(A_{1} \cap A_{2}\right)^{\prime}\right), \\
& \left(A_{1}, B_{1}\right) \sqcup\left(A_{2}, B_{2}\right):=\left(\left(B_{1} \cap B_{2}\right)^{\prime}, B_{1} \cap B_{2}\right), \\
& \left.\neg(A, B):=\left(A^{\prime}, A^{c^{\prime}}\right),\right\lrcorner(A, B):=\left(B^{c^{\prime}}, B^{c}\right), \top:=(G, \phi), \text { and } \perp:=(\phi, M) .
\end{aligned}
$$

Notation 3. $\mathfrak{P}(\mathbb{K})$ forms a abstract algebra of type $(2,2,1,1,0,0)$ with respect to the above operations and is called the protoconcept algebra of a context $\mathbb{K}$. It is denoted by $\mathfrak{P}(\mathbb{K}):=(\mathfrak{P}(\mathbb{K}), \sqcup, \sqcap, \neg\lrcorner,, \top, \perp)$.

$\mathfrak{H}(\mathbb{K})$ is closed under the above operations, forming a subalgebra of the protoconcept algebra. This algebra is called the semiconcept algebra of a context $\mathbb{K}$, and is denoted by $\underline{\mathfrak{H}}(\mathbb{K}):=(\mathfrak{H}(\mathbb{K}), \sqcup, \sqcap, \neg\lrcorner,, \top, \perp)$.

On abstraction of properties of the protoconcept algebra of a context, Wille defined the double Boolean algebra [11]. The semiconcept algebra leads to the notion of a pure double Boolean algebra.

Definition 3 [11]. A double Boolean algebra $(\mathrm{dBa})$ is an abstract algebra $\boldsymbol{D}:=$ $(D, \sqcup, \sqcap, \neg\lrcorner,, \top, \perp)$ which satisfies the following properties, for any $x, y, z \in D$.
(1a) $(x \sqcap x) \sqcap y=x \sqcap y$
(1b) $(x \sqcup x) \sqcup y=x \sqcup y$
(2a) $x \sqcap y=y \sqcap x$
(2b) $x \sqcup y=y \sqcup x$
(3a) $x \sqcap(y \sqcap z)=(x \sqcap y) \sqcap z$
(3b) $x \sqcup(y \sqcup z)=(x \sqcup y) \sqcup z$
(4a) $\neg(x \sqcap x)=\neg x$
(4b) $\lrcorner(x \sqcup x)=\lrcorner x$
(5a) $x \sqcap(x \sqcup y)=x \sqcap x$
(5b) $x \sqcup(x \sqcap y)=x \sqcup x$
(6a) $x \sqcap(y \vee z)=(x \sqcap y) \vee(x \sqcap z)$
(6b) $x \sqcup(y \wedge z)=(x \sqcup y) \wedge(x \sqcup z)$
(7a) $x \sqcap(x \vee y)=x \sqcap x$
(7b) $x \sqcup(x \wedge y)=x \sqcup x$
(8a) $\neg \neg(x \sqcap y)=x \sqcap y$
(9a) $x \sqcap \neg x=\perp$
(8b) $\lrcorner\lrcorner(x \sqcup y)=x \sqcup y$
(10a) $\neg \perp=\top \sqcap \top$
(9b) $x \sqcup\lrcorner x=\top$
(11a) $\neg \top=\perp$
(10b) $\lrcorner \top=\perp \sqcup \perp$
(11b) $\lrcorner \perp=\top$

$12(x \sqcap x) \sqcup(x \sqcap x)=(x \sqcup x) \sqcap(x \sqcup x)$,

where $x \vee y:=\neg(\neg x \sqcap \neg y)$ and $x \wedge y:=\lrcorner(\lrcorner x \sqcup\lrcorner y)$.

On $D$, a quasi-order $\sqsubseteq$ is given by $x \sqsubseteq y \Longleftrightarrow x \sqcap y=x \sqcap x$ and $x \sqcup y=y \sqcup y$, for any $x, y \in D$.

A dBa $\mathbf{D}$ is called pure, if for all $x \in D$ either $x \sqcap x=x$ or $x \sqcup x=x$.

Theorem 1 [1]]. $\mathfrak{P}(\mathbb{K})$ forms a $\mathrm{dBa}$ and $\underline{\mathfrak{H}}(\mathbb{K})$ forms a pure $\mathrm{dBa}$.

Notation 4. For any $\mathrm{dBa} \mathbf{D}:=(D, \sqcup, \sqcap, \neg\lrcorner,, \top, \perp), D_{\sqcap}:=\{x \in D \mid x \sqcap x=$ $x\}, D_{\sqcup}:=\{x \in D \mid x \sqcup x=x\}$. For $x \in D, x_{\sqcap}:=x \sqcap x$ and $x_{\sqcup}:=x \sqcup x$.

Let $\mathbf{D}:=(D, \sqcup, \sqcap, \neg\lrcorner,, \top, \perp)$ be a dBa. Then we have 


\section{Proposition $1[8]$.}

(i) $\boldsymbol{D}_{\sqcap}:=\left(D_{\sqcap}, \sqcap, \vee, \neg, \perp, \neg \perp\right)$ is a Boolean algebra, whose order relation is the restriction of $\sqsubseteq$ to $D_{\sqcap}$ and is denoted by $\sqsubseteq \sqcap$.

(ii) $\left.\left.\boldsymbol{D}_{\sqcup}:=\left(D_{\sqcup}, \sqcup, \wedge,\right\lrcorner, \top,\right\lrcorner \top\right)$ is a Boolean algebra, whose order relation is the restriction of $\sqsubseteq$ to $D \sqcup$ and is denoted by $\sqsubseteq \sqcup$.

(iii) $x \sqsubseteq y$ if and only if $x \sqcap x \sqsubseteq y \sqcap y$ and $x \sqcup x \sqsubseteq y \sqcup y$ for $x, y \in D$, that is $x_{\sqcap} \sqsubseteq \sqcap y_{\sqcap}$ and $x_{\sqcup} \sqsubseteq y_{\sqcup}$, for any $x, y \in D$.

The following proposition giving further properties of dBas is useful. Part (a) is proved in [7]; we prove (b) below.

\section{Proposition 2.}

(a) [7] For any $x, y, a \in D$ :

1. $x \sqcap \perp=\perp$ and $x \sqcup \perp=x \sqcup x$, that is $\perp \sqsubseteq x$.

2. $x \sqcup \top=\top$ and $x \sqcap \top=x \sqcap x$, that is $x \sqsubseteq \top$.

3. $x=y$ implies that $x \sqsubseteq y$ and $y \sqsubseteq x$.

4. $x \sqsubseteq y$ and $y \sqsubseteq x$ if and only if $x \sqcap x=y \sqcap y$ and $x \sqcup x=y \sqcup y$.

5. $x \sqcap y \sqsubseteq x, y \sqsubseteq x \sqcup y, x \sqcap y \sqsubseteq y, x \sqsubseteq x \sqcup y$.

6. $x \sqsubseteq y$ implies $x \sqcap a \sqsubseteq y \sqcap a$ and $x \sqcup a \sqsubseteq y \sqcup a$.

(b) For any $x, y \in D$ :

1. $\neg x=(\neg x)_{\sqcap} \in D_{\sqcap}$ and $\left.\lrcorner x=(\lrcorner x\right)_{\sqcup} \in D_{\sqcup}$.

2. $x \sqsubseteq y$ if and only if $\neg y \sqsubseteq \neg x$ and $\lrcorner y \sqsubseteq\lrcorner x$.

Proof. (b) 1. Let $x \in D$. By axiom (1a), $x \sqcap x \in D_{\sqcap}$. Using axiom (4a) and Proposition 1 (i) we have $\neg x=\neg(x \sqcap x) \in D_{\sqcap}$. The other is proved dually.

2. Let $x, y \in D$. Proposition 1 (iii) gives $x \sqsubseteq y$ if and only if $x_{\sqcap} \sqsubseteq \sqcap y_{\sqcap}$ and $x_{\sqcup} \sqsubseteq \sqcup$ $y \sqcup$, which is if and only if $\neg y_{\sqcap} \sqsubseteq \sqcap \neg x_{\sqcap}$ and $\left.\lrcorner y_{\sqcup} \sqsubseteq \sqcup\right\lrcorner x_{\sqcup}$ by Proposition 1(i)(ii). The latter is if and only if $\neg y \sqsubseteq \sqcap \neg x$ and $\lrcorner y \sqsubseteq \sqcup\lrcorner x$ (using axioms (4a) and (4b)), which is if and only if $\neg y \sqsubseteq \neg x$ and $\lrcorner y \sqsubseteq\lrcorner x$, by Proposition 1(i)-(ii) and (b)(1) of this Proposition.

Definition 4. Let $\mathbf{D}$ and $\mathbf{M}$ be two dBas. A map $h: M \rightarrow D$ is called a homomorphism if $h$ preserves the operations in the algebras.

$h$ is called quasi-injective, when $x \sqsubseteq y$ if and only if $h(x) \sqsubseteq h(y)$, for all $x, y \in M$. A quasi-injective and surjective homomorphism is called a quasiisomorphism and a bijective homomorphism is called an isomorphism.

In a dBa $\mathbf{D}:=(D, \sqcup, \sqcap, \neg\lrcorner,, \top, \perp)$ a subset $F$ of $D$ is called a filter, if it is an upset and closed under $\sqcap$. Dually, a subset $I$ of $D$ is called an ideal if it is a downset and closed under $\sqcup$.

$F_{0}(\subseteq D)$ is called a base for the filter $F$ if $F=\{y \in D: x \sqsubseteq y$ for some $x \in$ $\left.F_{0}\right\}$. Base for an ideal is dually defined.

Notation 5. Let $\mathbf{D}:=(D, \sqcup, \sqcap, \neg\lrcorner,, \top, \perp)$ be a dBa.

$\mathcal{F}_{p}(\mathbf{D}):=\left\{F \subseteq D \mid F\right.$ is a filter of $\mathbf{D}$ and $F \cap D_{\sqcap}$ is a prime filter in $\left.\mathbf{D}_{\sqcap}\right\}$, and $\mathcal{I}_{p}(\mathbf{D}):=\left\{I \subseteq D \mid I\right.$ is an ideal of $\mathbf{D}$ and $I \cap D_{\sqcup}$ is a prime ideal in $\left.\mathbf{D}_{\sqcup}\right\}$.

For any $x \in D, F_{x}:=\left\{F \in \mathcal{F}_{p}(\mathbf{D}) \mid x \in F\right\}$ and $I_{x}:=\left\{I \in \mathcal{I}_{p}(\mathbf{D}) \mid x \in I\right\}$.

Define the context $\mathbb{K}(\mathbf{D}):=\left(\mathcal{F}_{p}(\mathbf{D}), \mathcal{I}_{p}(\mathbf{D}), \Delta\right)$, where for any $F \in$ $\mathcal{F}_{p}(\mathbf{D}), I \in \mathcal{I}_{p}(\mathbf{D}), F \Delta I$ if and only if $F \cap I \neq \emptyset$. 
Lemma 1 [11]. Let $F$ be a filter of a dBa $\mathbf{D}$.

1. $F \cap D_{\sqcap}$ and $F \cap D_{\sqcup}$ are filters of the Boolean algebras $\boldsymbol{D}_{\sqcap}, \boldsymbol{D}_{\sqcup}$ respectively.

2. Each filter $F_{0}$ of the Boolean algebra $\boldsymbol{D}_{\sqcap}$ is the base of some filter $F$ of $\mathbf{D}$ such that $F_{0}=F \cap D_{\sqcap}$. Moreover if $F_{0}$ is a prime filter of $\boldsymbol{D}_{\sqcap}, F \in \mathcal{F}_{p}(\boldsymbol{D})$.

A similar result can be proved for ideals in a dBa.

Lemma 2. $\left(F_{x}\right)^{c}=F_{\neg x}$ and $\left(I_{x}\right)^{c}=I_{\lrcorner x}$, for any $x \in D$.

Theorem 2 [1]]. The map $h: D \rightarrow \mathfrak{P}(\mathbb{K}(\boldsymbol{D}))$ defined by $h(x):=\left(F_{x}, I_{x}\right)$ for all $x \in D$, is a quasi-injective homomorphism.

Theorem 3 [1]. If $\boldsymbol{D}:=(D, \sqcup, \sqcap, \neg\lrcorner,, \top, \perp)$ is a pure dBa, the map $h: D \rightarrow$ $\mathfrak{H}(\mathbb{K}(\boldsymbol{D}))$ defined by $h(x):=\left(F_{x}, I_{x}\right)$ for all $x \in D$, is an injective homomorphism.

\section{Concepts and Semiconcepts Based on Rough Set Theory}

In [4], Düntsch and Gediga pointed out limitations of FCA as a tool for qualitive data analysis. They gave the example of a context $\mathbb{K}:=(G, M, \Gamma)$, where $G$ is a set of problems and $M$ is a set of skills, and the relation $\Gamma \subseteq G \times M$ may be interpreted in two different ways:

I. Skill $s$ is necessary to solve $q$ and $\Gamma(q)$ is minimally sufficient to solve $q$.

II. It is possible to solve problem $q$ with skill $s$.

In this section, we work with an instance of such a context given below.

Example 1 Let $G:=\left\{q_{1}, q_{2}, q_{3}, q_{4}, q_{5}, q_{6}\right\}$ be a set of problems and consider a set of skills $S:=\left\{s_{1}, s_{2}, s_{3}, s_{4}, s_{5}, s_{6}, s_{7}, s_{8}, s_{9}, s_{10}, s_{11}\right\}$. The context $\mathbb{K}:=(G, M, \Gamma)$ is represented by the table below. A cross in the $i-j$-th cell of the table indicates that the relation $q_{i} \Gamma s_{j}$ holds.

Table 1. Context

\begin{tabular}{l|l|l|l|l|l|l|l|l|l|l|l}
\hline & $s_{1}$ & $s_{2}$ & $s_{3}$ & $s_{4}$ & $s_{5}$ & $s_{6}$ & $s_{7}$ & $s_{8}$ & $s_{9}$ & $s_{10}$ & $s_{11}$ \\
\hline$q_{1}$ & $\times$ & & $\times$ & & $\times$ & & $\times$ & & & $\times$ & \\
\hline$q_{2}$ & & $\times$ & $\times$ & $\times$ & & $\times$ & & $\times$ & & & \\
\hline$q_{3}$ & $\times$ & $\times$ & & $\times$ & & $\times$ & & & $\times$ & & \\
\hline$q_{4}$ & $\times$ & & $\times$ & & $\times$ & & & & & & \\
\hline$q_{5}$ & & & & & $\times$ & & & $\times$ & $\times$ & & $\times$ \\
\hline$q_{6}$ & & & & & & & & & & & $\times$ \\
\hline
\end{tabular}


Case I: Let $q_{i} \Gamma s_{j}$ be interpreted as: skill $s_{j}$ is necessary to solve $q_{i}$, and $\Gamma\left(q_{i}\right)$ is minimally sufficient to solve $q_{i}$. Let a student be asked to solve a set of problems $A:=\left\{q_{1}, q_{2}\right\}$ in a test to check his skills (from the set $S$ ). In FCA, $A^{\prime}=\left\{s_{3}\right\}$ gives the collection of the skills necessary to solve all the problems of $A$. However, different problems in $A$ may require different sets of skills to solve them. For instance, $q_{1}$ requires $s_{1}$ while $q_{2}$ does not. So $\left\{s_{1}, s_{2}, s_{3}, s_{4}, s_{5}, s_{6}, s_{7}, s_{8}, s_{10}\right\}$ would more adequately represent the skills necessary to solve $A$.

Case II: Let us interpret $q_{i} \Gamma s_{j}$ as: it is possible to solve $q_{i}$ with skill $s_{j}$. Considering the problem set $A:=\left\{q_{1}, q_{2}\right\}$, again $A^{\prime}=\left\{s_{3}\right\}$ does not give all the possible skills that could be used to solve the problems in $A$ - for instance, it is possible to solve them with the skills $s_{1}$ and $s_{2}$.

Now the question is, how do we assign a skill set to the problem set $A$ ? Düntsch and Gediga address this question in [4], using modal style operators and introduce property oriented concepts. Let $\mathbb{K}:=(G, M, R)$ be a context, $A \subseteq G$ and $B \subseteq M$.

$$
\begin{gathered}
B_{R}^{\diamond}:=\{x \in G \mid R(x) \cap B \neq \emptyset\} \text { and } B_{R}^{\square}:=\{x \in G \mid R(x) \subseteq B\} \\
A_{R^{-1}}^{\diamond}:=\left\{y \in M \mid R^{-1}(y) \cap A \neq \emptyset\right\} \text { and } A_{R^{-1}}^{\square}:=\left\{y \in M \mid R^{-1}(y) \subseteq A\right\}
\end{gathered}
$$

If there is no confusion about the relation involved, we shall omit the subscript and denote $B_{R}^{\diamond}$ by $B^{\diamond}, B_{R}^{\square}$ by $B^{\square}$, and similarly for the case of $A$.

Definition 5 [3]. A closure operator on a set $X$ is an operator $C$ on the power set $\mathcal{P}(X)$ of $X$ such that for all $A, B \in \mathcal{P}(X)$,

C1 $A \subseteq C(A)$,

$\mathrm{C} 2 A \subseteq B$ implies $C(A) \subseteq C(B)$,

C3 $C(C(A))=C(A)$.

$A \in \mathcal{P}(X)$ is called closed if and only if $C(A)=A$.

An interior operator $I$ on the set $X$ is defined dually, and $A \in \mathcal{P}(X)$ is called open if and only if $I(A)=A$.

Some properties of the operators $\square, \diamond$ are as follows.

Theorem 4 [14]. Let $A, A_{1}, A_{2} \subseteq G$ and $B, B_{1}, B_{2} \subseteq M$.

1. $A_{1} \subseteq A_{2}$ implies that $A_{1}^{\square} \subseteq A_{2}^{\square}$ and $A_{1}^{\diamond} \subseteq A_{2}^{\diamond}$.

2. $B_{1} \subseteq B_{2}$ implies that $B_{1}^{\square} \subseteq B_{2}^{\square}$ and $B_{1}^{\diamond} \subseteq B_{2}^{\diamond}$.

3. $A_{R}^{\square}=A_{-R}^{c \prime} ; B_{R}^{\square}=B_{-R}^{c \prime}$ and $A_{R}^{\diamond}=A_{-R}^{\prime c} ; B_{R}^{\diamond}=B_{-R}^{\prime c}$.

4. $A^{\square \diamond \square}=A^{\square}$ and $B^{\square \diamond \square}=B^{\square}$.

5. $A^{\diamond \square \diamond}=A^{\diamond}$ and $B^{\diamond \square \diamond}=B^{\diamond}$.

6. $\square \diamond$ is interior operator on $G$ and $\diamond \square$ is closure operator on $M$.

Let $\mathbb{K}:=(G, M, R)$ be a context, $A \subseteq G, B \subseteq M$. 
Definition $6[4] \cdot(A, B)$ is a property oriented concept of $\mathbb{K}$ if it satisfies the conditions $A^{\diamond}=B$ and $B^{\square}=A$.

Refer to Example 1, Case I. For any $A \subseteq G, A^{\diamond}$ can be interpreted as the set of all skills $m$ that are necessary to solve problems in $A\left(\Gamma^{-1}(m) \cap A \neq \emptyset\right)$. For any $B \subseteq M, B^{\square}$ can be interpreted as the set of problems $x$ such that $B$ contains all skills sufficient to solve $x(\Gamma(x) \subseteq B)$. So the intent $B$ of a property oriented concept $(A, B)$ of the context given in Example 1, represents the set of skills that are necessary and sufficient to solve problems in the extent $A$. For instance, $\left\{s_{1}, s_{2}, s_{3}, s_{4}, s_{5}, s_{6}, s_{7}, s_{8}, s_{10}\right\}$ gives all the skills that are necessary and sufficient to solve the problems in $\left\{q_{1}, q_{2}, q_{4}\right\}$; hence $\left(\left\{q_{1}, q_{2}, q_{4}\right\},\left\{s_{1}, s_{2}, s_{3}, s_{4}, s_{5}, s_{6}, s_{7}, s_{8}, s_{10}\right\}\right)$ is a property-oriented concept.

Definition $7[13] . \quad(A, B)$ is an object oriented concept of the context $\mathbb{K}$ if it satisfies the condition $A^{\square}=B$ and $B^{\diamond}=A$.

An order relation $\leq$ can be defined on the set of such pairs. For any object oriented concepts $\left(A_{1}, B_{1}\right),\left(A_{2}, B_{2}\right),\left(A_{1}, B_{1}\right) \leq\left(A_{2}, B_{2}\right)$ if and only if $A_{1} \subseteq$ $A_{2}$ (equivalently, $B_{1} \subseteq B_{2}$ ).

Refer to Example 1, Case II. For $A \subseteq G, A^{\square}$ can be interpreted as the set of skills $s$ such that $A$ contains all the problems that are possible to solve with $s$ $\left(\Gamma^{-1}(s) \subseteq A\right)$. For $B \subseteq M, B^{\diamond}$ is the set of problems that are possible to solve with some skill in $B(\Gamma(x) \cap B \neq \emptyset)$. So the extent $A$ of an object oriented concept $(A, B)$ in this context is the set of problems which are possible to solve with skills in $B$, while the intent $B$ is the set of skills by which only problems in $A$ can be solved. $\left(\left\{q_{3}, q_{5}\right\},\left\{s_{9}\right\}\right)$ then forms an object-oriented concept.

A comparative study of Wille's concepts, property and object oriented concepts and concept lattices is done extensively by Yao in [13].

We next turn to object-oriented semiconcepts, introduced in [6] to bring in the notion of negation.

Definition $8[6] .(A, B)$ is an object oriented semiconcept of $\mathbb{K}$ if $A^{\square}=B$ or $B^{\diamond}=A$.

Notation 6. The set of all object oriented concepts is denoted by $\mathbf{R O}-\mathbf{L}(\mathbb{K})$, the set of all object oriented semiconcepts is denoted by $\mathfrak{S}(\mathbb{K})$.

The following are observed in [6].

\section{Observation 2.}

1. $(A, B) \in \mathfrak{S}(\mathbb{K})$ if and only if either $(A, B)=\left(A, A^{\square}\right)$ or $(A, B)=\left(B^{\diamond}, B\right)$.

2. $R O-L(\mathbb{K}) \subseteq \mathfrak{S}(\mathbb{K})$.

3. $(A, B)$ is a semiconcept of $\mathbb{K}$ if and only if $\left(A^{c}, B\right)$ is an object oriented semiconcept of the context $\mathbb{K}^{c}$. 
The following operations are defined in $\mathfrak{S}(\mathbb{K})$. Let $\left(A_{1}, B_{1}\right),\left(A_{2}, B_{2}\right),(A, B)$ be any object oriented semiconcepts.

$$
\begin{aligned}
& \left(A_{1}, B_{1}\right) \sqcap\left(A_{2}, B_{2}\right):=\left(\left(B_{1} \cap B_{2}\right)^{\diamond}, B_{1} \cap B_{2}\right), \\
& \left(A_{1}, B_{1}\right) \sqcup\left(A_{2}, B_{2}\right):=\left(A_{1} \cup A_{2},\left(A_{1} \cup A_{2}\right)^{\square}\right), \\
& \left.\neg(A, B):=\left(A^{c}, A^{c \square}\right),\right\lrcorner(A, B):=\left(B^{c \diamond}, B^{c}\right), \top:=(G, M), \text { and } \perp:=(\phi, \phi) .
\end{aligned}
$$

Refer again to Example 1, Case II. It is clear that it is possible to solve the problems $q_{1}, q_{2}, q_{4}$ with skills other than $s_{9}$. We are able to express this observation in the framework of object oriented semiconcepts. Note that $a:=\left(\left\{q_{3}, q_{5}\right\},\left\{s_{9}\right\}\right)$ is an object oriented semiconcept of the context. The extent of $a$ gives exactly the problems that are possible to solve with skill $s_{9}$. The negation of $\left.a,\right\lrcorner a$, is the object oriented semiconcept $\left(\left\{q_{1}, q_{2}, q_{3}, q_{4}, q_{5}, q_{6}\right\},\left\{s_{9}\right\}^{c}\right)$ whose extent gives all the problems that are possible to solve with skills other than $s_{9}$, and includes $q_{1}, q_{2}, q_{4}$.

In this work, for the sake of simplicity in expressions of results, we consider operations on object oriented semiconcepts that are dual to the ones mentioned above. In other words, for $(A, B),(C, D) \in \mathfrak{S}(\mathbb{K})$, we consider

$$
\begin{aligned}
& (A, B) \sqcap(C, D):=\left(A \cup C,(A \cup C)^{\square}\right), \\
& (A, B) \sqcup(C, D):=\left((B \cap D)^{\diamond}, B \cap D\right), \\
& \lrcorner(A, B):=\left(B^{c \diamond}, B^{c}\right), \neg(A, B):=\left(A^{c}, A^{c \square}\right), \top:=(\emptyset, \emptyset), \perp:=(G, M) .
\end{aligned}
$$

From the results established in [6], we obtain

\section{Theorem 5.}

1. $\underline{\mathcal{S}}(\mathbb{K}):=(\mathfrak{S}(\mathbb{K}), \sqcup, \sqcap, \neg\lrcorner,, \top, \perp)$ is a pure dBa.

2. $\underline{\mathfrak{H}}(\mathbb{K})$ is isomorphic to $\underline{\mathcal{S}}\left(\mathbb{K}^{c}\right)$.

The map in (2) of the above theorem is due to Observation 2(3). From Lemma 2, Theorem 5(2) and Theorem 3 we have the following.

Theorem 6. For a pure dBa $\mathbf{D}$ the map $h: D \rightarrow \mathfrak{S}\left(\mathbb{K}^{c}(\mathbf{D})\right)$ defined by $h(x):=$ $\left(F_{\neg x}, I_{x}\right)$ for all $x \in D$, is an injective $\mathrm{dBa}$ homomorphism.

\section{Object Oriented Protoconcepts}

We now define and give some properties of object oriented protoconcepts. As before, $\mathbb{K}:=(G, M, R)$ is a context, $A \subseteq G, B \subseteq M$.

Definition 9. $(A, B)$ is an object oriented protoconcept of $\mathbb{K}$ if $A^{\square \diamond}=B^{\diamond}$.

Notation 7. The set of all object oriented protoconcepts is denoted by $\mathfrak{R}(\mathbb{K})$.

\section{Proposition 3.}

1. $A^{\square \diamond}=B^{\diamond}$ if and only if $A^{\square}=B^{\diamond \square}$. 
2. $\mathfrak{S}(\mathbb{K}) \subseteq \mathfrak{R}(\mathbb{K})$.

Proof. (1) Let $A^{\square \diamond}=B^{\diamond}$. Then $A^{\square \diamond \square}=B^{\diamond \square}$. Now from 4 of Theorem 4 we know that $A^{\square \diamond \square}=A^{\square}$. Therefore $A^{\square}=B^{\diamond \square}$. Let $A^{\square}=B^{\diamond \square}$ then $A^{\square \diamond}=$

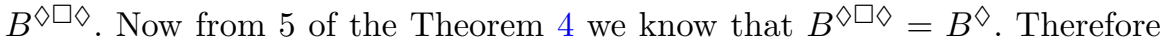
$A^{\square \diamond}=B^{\diamond}$.

(2) Let $(A, B) \in \mathfrak{S}(\mathbb{K})$ then either $A^{\square}=B$ or $B^{\diamond}=A$. Now if $A^{\square}=B$ then we gate $A^{\square}=B^{\diamond}$ and hence $(A, B) \in \mathfrak{R}(\mathbb{K})$. Now if $B^{\diamond}=A$ then $A^{\square}=B^{\diamond \square}$. Therefore $(A, B) \in \mathfrak{R}(\mathbb{K})$ by $(1)$ of this Proposition. Hence $\mathfrak{S}(\mathbb{K}) \subseteq \mathfrak{R}(\mathbb{K})$.

Recall Example 1 and Case II. Consider $A_{1}:=\left\{q_{1}, q_{2}, q_{4}, q_{6}\right\}$ and $B_{1}:=\left\{s_{3}\right\}$. Then $A_{1}^{\square \diamond}=B_{1}^{\diamond}$, so that $\left(A_{1}, B_{1}\right)$ is an object oriented protoconcept of the context. Now observe that $B_{1}^{\diamond}=\left\{q_{1}, q_{2}, q_{4}\right\}$ and so $B_{1}^{\diamond} \neq A_{1} ; A_{1}^{\square}=\left\{s_{3}, s_{7}, s_{10}\right\}$, so $A_{1}^{\square} \neq B_{1}$. This means $\left(A_{1}, B_{1}\right) \in \mathfrak{R}(\mathbb{K})$ but $\left(A_{1}, B_{1}\right) \notin \mathfrak{S}(\mathbb{K})$, indicating that the converse of (2) in Proposition 3 is not true.

We next characterize the object oriented protoconcepts of $\mathbb{K}$ (Theorem 7 below) using a notion of 'approximation' by object oriented concepts. Based on the facts that $\square \diamond$ is an interior operator on $G$ and $\diamond \square$ is a closure operator on $M$, the discussion in [15] of 'definable' object sets gives the definition below.

\section{Definition 10.}

1. $A$ is said to be definable in $\mathcal{P}(G)$ if and only if $A^{\square \diamond}=A$.

2. $B$ is said to be definable in $\mathcal{P}(M)$ if and only if $B^{\diamond \square}=B$.

It is then easy to see the following.

Proposition 4. $A$ is definable if and only if it is the extent of some object oriented concept of $\mathbb{K}$, and $B$ is definable if and only if it is the intent of some object-oriented concept of $\mathbb{K}$.

Proof. From Definition 7 it follows that for any object oriented concept $(A, B)$, $A, B$ are definable. To see the converse assertions, for definable $A$ and $B$, consider respectively the object oriented concepts $\left(A, A^{\square}\right)$ and $\left(B^{\diamond}, B\right)$.

So an object oriented concept $(A, B)$ of the context $\mathbb{K}$ can be thought of as a pair of definable sets such that one can be determined by the other as $A=B^{\diamond}$ and $A^{\square}=B$. However, for pairs $(E, F) \in \mathcal{P}(G) \times \mathcal{P}(M)$ which are not object oriented concepts, it may be worthwhile to determine the largest definable set $C$ contained in $E$ and determined by $F$ as $C=F^{\diamond}$, and also the smallest definable set $D$ containing $F$ and determined by $E$ as $E^{\square}=D$. Such a pair $(C, D)$ would be an object oriented concept, and unique in the above respect. For instance, referring to Example 1, Case II: for a set $E$ of problems and set $F$ of skills, $C$ would be the largest definable set of problems inside $E$ that are possible to be solved with the skills in $F\left(C=F^{\diamond}\right)$. We call the pair $(C, D)$ an approximation of the pair $(E, F)$.

Definition 11. An object oriented concept $(C, D)$ is called an approximation of $(A, B)$ if and only if $C$ is the largest definable set in $\mathcal{P}(G)$ contained in $A$, and $D$ is the smallest definable set in $\mathcal{P}(M)$ containing $B$. 
Observation 3. If $(A, B)$ has an approximation, it is unique.

Theorem 7. $(A, B)$ is an object oriented protoconcept of $\mathbb{K}$ if and only if it has an approximation.

Proof. Let $(A, B)$ be an object oriented protoconcept of $\mathbb{K}$. Then $A^{\square \diamond}=B^{\diamond}$, which is equivalent to $A^{\square}=B^{\diamond \square}$. Now we set $C:=A^{\square \diamond}$ and $D:=A^{\square}=B^{\diamond \square}$. Then the pair $(C, D)=\left(A^{\square}, A^{\square}\right)$ is an object oriented concept. Since $\square \diamond$ is an interior operator on $G, C \subseteq A$; since $\diamond \square$ is a closure operator on $M, B \subseteq D$. As $C$ is the extent of the object oriented concept $(C, D), C$ is definable in $\mathcal{P}(G)$ by Proposition 4. Now let $E$ be a definable set in $\mathcal{P}(G)$ such that $E \subseteq A$. By 1 of Theorem 4 we have $E=E^{\square \diamond} \subseteq A^{\square \diamond}=C$, making $C$ the largest definable set contained in $A$. Similarly we get that $D$ is the smallest definable set containing $B$. So $(C, D)$ is an approximation of $(A, B)$.

For the converse, let us assume that $(X, Y)$ is an approximation of $(A, B)$. Then $(X, Y)$ is an object oriented concept and $X \subseteq A$ and $B \subseteq Y$. Using 1 and 2 of Theorem 4 we have $X=X^{\square \diamond} \subseteq A^{\square \diamond}$ and $B^{\diamond \square} \subseteq Y^{\diamond \square}=Y$. By 4 and 6 of Theorem $4, A^{\square \diamond \square \diamond}=A^{\square \diamond} \subseteq A$, making $A^{\square \diamond}$ a definable set contained in $A$. Since $X$ is the largest definable set contained in $A, A^{\square \diamond} \subseteq X$. Dually we can show that $Y \subseteq B^{\diamond \square}$. So $X=A^{\square \diamond}$ and $Y=B^{\diamond \square}$. As $(X, Y)$ is an object oriented concept then $X^{\square}=Y$ and $X=Y^{\diamond}$. So $A^{\square}=A^{\square \diamond \square}=B^{\diamond \square}$ and hence $(A, B)$ is a object oriented protoconcept of $\mathbb{K}$.

In Example 1, Case II: as shown earlier, the pair $\left(\left\{q_{1}, q_{2}, q_{4}, q_{6}\right\},\left\{s_{3}\right\}\right)$ is an object oriented protoconcept but not an object oriented concept. This pair has the object oriented concept $\left(\left\{q_{1}, q_{2}, q_{4}\right\},\left\{s_{3}, s_{7}, s_{10}\right\}\right)$ as its unique approximation.

Proposition 5. $(A, B)$ is an object oriented protoconcept of $\mathbb{K}$ if and only if $\left(A^{c}, B\right)$ is a protoconcept of $\mathbb{K}^{c}$.

Proof. $A_{R}^{\square \diamond}=B_{R}^{\diamond}$ if and only if $A_{-R}^{c \prime \prime c}=B_{-R}^{\prime c}$ by 3 of Theorem 4 , and $A_{-R}^{c / \prime c}=B_{-R}^{\prime c}$ if and only if $A_{-R}^{c \prime \prime}=B_{-R}^{\prime}$.

Recall the operations $\sqcup, \sqcap, \neg,\lrcorner, \top, \perp$ defined in Sect. 3 that made the set $\mathfrak{S}(\mathbb{K})$ of object oriented semiconcepts a pure dBa (Theorem 5 ). The set $\mathfrak{R}(\mathbb{K})$ of object oriented protoconcepts turns out to be closed with respect to the same operations. In fact,

Theorem 8. (i) $\mathfrak{R}(\mathbb{K}):=(\mathfrak{R}(\mathbb{K}), \sqcup, \sqcap, \neg\lrcorner,, \top, \perp)$ is a dBa and (ii) $\mathfrak{R}(\mathbb{K})$ is isomorphic to $\left.\left(\mathfrak{P}\left(\mathbb{K}^{c}\right), \sqcup, \sqcap, \neg,\right\lrcorner, \top, \perp\right)$.

Proof. (i) The proof is a routine check.

(ii) $h: \mathfrak{R}(\mathbb{K}) \rightarrow \mathfrak{P}\left(\mathbb{K}^{c}\right)$ defined as $h((A, B)):=\left(A^{c}, B\right)$ for any $(A, B)$ in $\mathfrak{R}(\mathbb{K})$, is an isomorphism between the two dBas. $h$ is well defined and onto by Proposition 5. Injectivity of $h$ follows trivially. Verifying that $h$ is a homomorphism is a routine check.

The $\mathrm{dBa} \underline{\Re}(\mathbb{K})$ is called the algebra of object oriented protoconcepts. 
Observation 4. Using the definition of the quasi-order in a $\mathrm{dBa}$, one sees that for any $(A, B),(C, D) \in \mathfrak{R}(\mathbb{K}),(A, B) \sqsubseteq(C, D)$ if and only if $C \subseteq A$ and $D \subseteq B$.

Using Theorems 2 and 8 and Lemma 2, we get a representation result for dBas.

Theorem 9. For a dBa D, the map $h: D \rightarrow \mathfrak{R}\left(\mathbb{K}^{c}(\mathbf{D})\right)$ defined by $h(x):=$ $\left(F_{\neg x}, I_{x}\right)$ for any $x \in D$, is a quasi-injective $\mathrm{dBa}$ homomorphism.

\section{Logics for dBas and Pure dBas}

We now give a sequent calculus DBL for the class of dBas, and extend it to PDBL to get a sequent calculus for pure dBas. The language of DBL consists of propositional constants $\perp, \top$, a set VAR of propositional variables, and logical connectives $\sqcup, \sqcap, \neg$,$\lrcorner . The set \mathfrak{F}$ of all formulae of DBL is given by the scheme:

$$
\perp|\top| p|\alpha \sqcup \beta| \alpha \sqcap \beta|\neg \alpha|\lrcorner \alpha,
$$

where $p \in$ VAR. $\vee$ and $\wedge$ are definable connectives: $\alpha \vee \beta:=\neg(\neg \alpha \sqcap \neg \beta)$ and $\alpha \wedge \beta:=\lrcorner(\lrcorner \alpha \sqcup\lrcorner \beta)$, for $\alpha, \beta \in \mathfrak{F}$.

A sequent in DBL with formulae $\alpha, \beta \in \mathfrak{F}$ is denoted in the usual manner as $\alpha \vdash \beta$. $\alpha \dashv \beta$ will be used as abbreviation for $(\alpha \vdash \beta$ and $\beta \vdash \alpha)$.

The axioms of DBL are given by the following schemes.
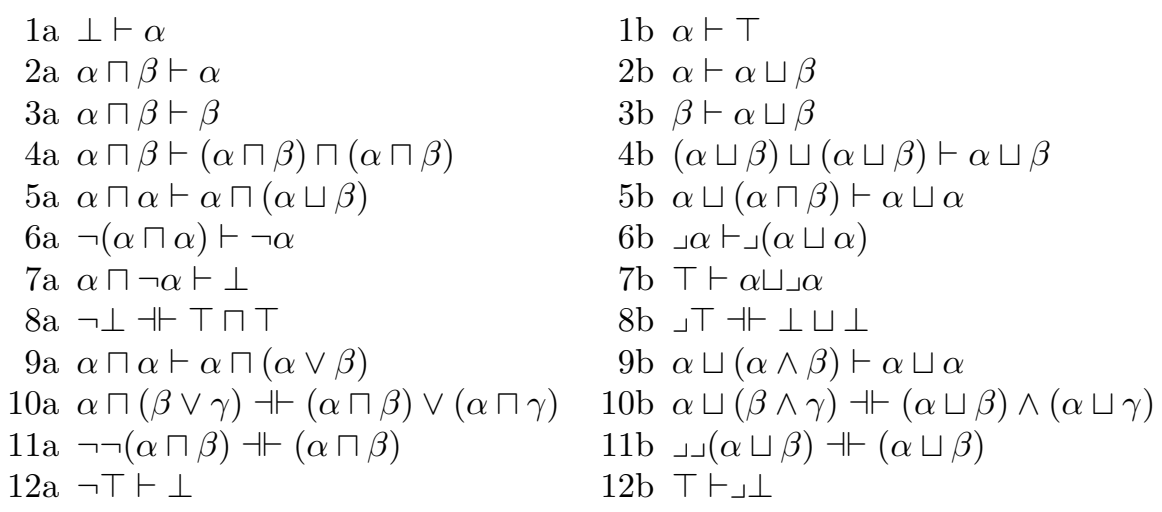

$13 \alpha \vdash \alpha$

$14(\alpha \sqcup \alpha) \sqcap(\alpha \sqcup \alpha) \vdash(\alpha \sqcap \alpha) \sqcup(\alpha \sqcap \alpha)$ 
Rules of inference:

$$
\begin{gathered}
\frac{\alpha \vdash \beta}{\alpha \sqcap \gamma \vdash \beta \sqcap \gamma}(R 1) \\
\frac{\alpha \vdash \beta}{\alpha \sqcup \gamma \vdash \beta \sqcup \gamma}(R 2) \\
\frac{\alpha \vdash \beta}{\neg \beta \vdash \neg \alpha}(R 3) \\
\frac{\alpha \vdash \beta, \beta \vdash \gamma}{\alpha \vdash \gamma}(R 4)
\end{gathered}
$$

$$
\begin{gathered}
\frac{\alpha \vdash \beta}{\gamma \sqcap \alpha \vdash \gamma \sqcap \beta}\left(R 1^{\prime}\right) \\
\frac{\alpha \vdash \beta}{\gamma \sqcup \alpha \vdash \gamma \sqcup \beta}(R 2)^{\prime} \\
\frac{\alpha \vdash \beta}{\lrcorner \beta \vdash\lrcorner \alpha}(R 5) \\
\frac{\alpha \sqcap \beta \dashv \alpha \sqcap \alpha \quad \alpha \sqcup \beta \dashv \beta \sqcup \beta}{\alpha \vdash \beta}(R 6)
\end{gathered}
$$

Provability of sequents in DBL is defined in the standard way.

Proposition 6. The following rules are derivable in DBL.

$$
\frac{\alpha \vdash \beta \quad \alpha \vdash \gamma}{\alpha \sqcap \alpha \vdash \beta \sqcap \gamma}(R 7) \quad \frac{\beta \vdash \alpha \gamma \vdash \alpha}{\beta \sqcup \gamma \vdash \alpha \sqcup \alpha}(R 8)
$$

Proof. $(R 7)$ is derived using $(R 1),\left(R 1^{\prime}\right)$ and $(R 4)$, while for $(R 8)$ one uses $(R 2),\left(R 2^{\prime}\right)$ and $(R 4)$.

Theorem 10. The following sequents are provable in DBL.
(1a) $(\alpha \sqcap \beta) \dashv(\beta \sqcap \alpha)$.
(1b) $\alpha \sqcup \beta \dashv \beta \sqcup \alpha$.
(2a) $\alpha \sqcap(\beta \sqcap \gamma) \dashv(\alpha \sqcap \beta) \sqcap \gamma$.
(2b) $\alpha \sqcup(\beta \sqcup \gamma) \dashv(\alpha \sqcup \beta) \sqcup \gamma$.
(3a) $(\alpha \sqcap \alpha) \sqcap \beta \dashv(\alpha \sqcap \beta)$.
(3b) $(\alpha \sqcup \alpha) \sqcup \beta \dashv-\alpha \sqcup \beta$.
(4a) $\neg \alpha \vdash \neg(\alpha \sqcap \alpha)$.
(4b) $\lrcorner(\alpha \sqcup \alpha) \vdash\lrcorner \alpha$.
(5a) $\alpha \sqcap(\alpha \sqcup \beta) \vdash(\alpha \sqcap \alpha)$.
(5b) $\alpha \sqcup \alpha \vdash \alpha \sqcup(\alpha \sqcap \beta)$.
(6a) $\alpha \sqcap(\alpha \vee \beta) \vdash \alpha \sqcap \alpha$.
(6b) $\alpha \sqcup \alpha \vdash \alpha \sqcup(\alpha \wedge \beta)$.
(7a) $\perp \vdash \alpha \sqcap \neg \alpha$.
(7b) $\alpha \sqcup\lrcorner \alpha \vdash \top$.
(8a) $\perp \vdash \neg \top$
(8b) $\lrcorner \perp \vdash \top$.
(9) $(\alpha \sqcap \alpha) \sqcup(\alpha \sqcap \alpha) \vdash(\alpha \sqcup \alpha) \sqcap(\alpha \sqcup \alpha)$.

Proof. The proofs are straightforward. For instance, $(4 a)$ follows from axiom (2a) and $(R 3)$. The proofs of $(i b)$ are dual to those of $(i a)$ for $i=1,2,3,4,5,6$. $(7 a),(8 a)$ follow from axiom (1a), and $7 b, 8 b$ follow from axiom (1b). Proposition 6 is also used in some of the proofs.

PDBL is the logic obtained from DBL by adding the following axiom:

15. for any $\alpha \in \mathfrak{F}$, either $\alpha \vdash \alpha \sqcap \alpha$ or $\alpha \sqcup \alpha \vdash \alpha$.

Due to axioms $(2 \mathrm{a})$ and $(2 \mathrm{~b})$, we get

Proposition 7. In PDBL, for any $\alpha \in \mathfrak{F}$, either $\alpha \dashv-\alpha \sqcap \alpha$ or $\alpha \dashv-\alpha \sqcup \alpha$.

Now we define the notion of validity for DBL (PDBL) with respect to the class of dBas (pure dBas).

Definition 12. Let $\mathbf{D}$ be a (pure) dBa. A valuation $v: \mathfrak{F} \rightarrow D$ in $\mathbf{D}$ is a map such that for all $\alpha, \beta \in \mathfrak{F}$ we have the following. 

1. $v(\alpha \sqcup \beta):=v(\alpha) \sqcup v(\beta)$.
4. $v(\alpha \sqcap \beta):=v(\alpha) \sqcap v(\beta)$.
2. $v(\lrcorner \alpha):=\lrcorner v(\alpha)$.
5. $v(\neg \alpha):=\neg v(\alpha)$.
3. $v(\top):=\top$.
6. $v(\perp):=\perp$.

Definition 13. A sequent $\alpha \vdash \beta$ is said to be satisfied by a valuation $v$ in a (pure) dBa $\mathbf{D}$ if and only if $v(\alpha) \sqsubseteq v(\beta)$. A sequent $\alpha \vdash \beta$ is true in $\mathbf{D}$ if and only if for all valuations $v$ in $\mathbf{D}, v$ satisfies $\alpha \vdash \beta$. A sequent $\alpha \vdash \beta$ is valid in the class of all (pure) dBas if and only if it is true in every (pure) dBa.

Theorem 11 (Soundness). If $\alpha \vdash \beta$ is provable in DBL (PDBL) then it is valid in the class of all dBas (pure dBas).

Proof. The proof that all the axioms of DBL are valid in the class of all dBas and that the rules of inference preserve validity, is straightforward. Proposition 2 giving properties of dBas, is utilized. The result applies to PDBL and pure dBas, as axiom (15) reflects the defining axiom of pure dBas (Definition 3).

The completeness theorem is established in the standard way, using the Lindenbaum-Tarski algebras of the logics DBL and PDBL. We sketch the route taken by the proof. For $\alpha, \beta \in \mathfrak{F}$, a relation $\equiv_{\vdash}$ is defined on $\mathfrak{F}$ by: $\alpha \equiv \vdash \beta$ if and only if $\alpha \dashv \vdash \beta$. $\equiv \vdash$ is shown to be a congruence relation on $\mathfrak{F}$ with respect to $\sqcup, \sqcap, \neg$,$\lrcorner . The quotient set \mathfrak{F} / \equiv_{\vdash}$ induced by the relation $\equiv_{\vdash}$ and operations induced by the logical connectives, give the Lindenbaum-Tarski algebra $\left.\mathcal{L}(\mathfrak{F}):=\left(\mathfrak{F} / \equiv_{\vdash}, \sqcup, \sqcap, \neg,\right\lrcorner,[\top],[\perp]\right)$. The axioms in DBL (PDBL) show that $\mathcal{L}(\mathfrak{F})$ of the respective logic is a $\mathrm{dBa}$ (pure $\mathrm{dBa})$. One can then establish

Proposition 8. The following statements are equivalent.

1. $\alpha \vdash \beta$ is provable in DBL.

2. $[\alpha] \sqsubseteq[\beta]$ in $\mathcal{L}(\mathfrak{F})$ of $\mathbf{D B L}$.

The result can be extended to the case of PDBL. Using these and the canonical map $v: \mathfrak{F} \rightarrow \mathfrak{F} / \equiv \vdash$ defined as $v(\gamma):=[\gamma]$ for any $\gamma \in \mathfrak{F}$, one obtains

Theorem 12 (Completeness). If a sequent $\alpha \vdash \beta$ is valid in the class of all dBas (pure dBas) then it is provable in DBL (PDBL).

\section{Object Oriented Protoconcept and Semiconcept Semantics for the Logics}

In this section, we define object oriented protoconcept semantics for DBL and object oriented semiconcept semantics for PDBL, and show that the logics are sound and complete with respect to these semantics.

Definition 14. A model for $\boldsymbol{D B L}$ is a pair $\mathbb{M}:=(\mathbb{K}, v)$, where $\mathbb{K}$ is a context and $v$ is a map from the set $\mathfrak{F}$ of $\mathbf{D B L}$-formulae to the set $\mathfrak{R}(\mathbb{K})$ of all object oriented protoconcepts of $\mathbb{K}$ satisfying the following conditions: 

1. $v(\alpha \sqcup \beta):=v(\alpha) \sqcup v(\beta)$.
4. $v(\alpha \sqcap \beta):=v(\alpha) \sqcap v(\beta)$.
2. $v(\lrcorner \alpha):=\lrcorner v(\alpha)$.
5. $v(\neg \alpha):=\neg v(\alpha)$.
3. $v(\top):=\top$.
6. $v(\perp):=\perp$.

$\mathbb{M}:=(\mathbb{K}, v)$ is a model for PDBL if $v$ is a map from $\mathfrak{F}$ to the set $\mathfrak{S}(\mathbb{K})$ of all object oriented semiconcepts of $\mathbb{K}$. The properties satisfied by $v$ remain the same as above.

Notation 8. For the class $\mathcal{K}$ of all contexts, we define $\mathfrak{R}(\mathcal{K}):=\{\Re(\mathbb{K}) \mid \mathbb{K} \in \mathcal{K}\}$ and $\mathfrak{S}(\mathcal{K}):=\{\mathfrak{S}(\mathbb{K}) \mid \mathbb{K} \in \mathcal{K}\}$.

Definition 15. A sequent $\alpha \vdash \beta$ is said to be satisfied in a model $\mathbb{M}$ for DBL if and only if $v(\alpha) \sqsubseteq v(\beta)$ in $\mathfrak{R}(\mathbb{K})$. A sequent $\alpha \vdash \beta$ is true in $\mathfrak{R}(\mathbb{K})$ of a context $\mathbb{K}$ if and only if every model $\mathbb{M}$ based on the context $\mathbb{K}$ satisfies the sequent $\alpha \vdash \beta$. A sequent $\alpha \vdash \beta$ is valid in $\mathfrak{R}(\mathcal{K})$ if and only if it is true in every $\mathfrak{R}(\mathbb{K})$ of $\mathfrak{R}(\mathcal{K})$.

Replacing $\mathfrak{R}(\mathbb{K})$ and $\mathfrak{R}(\mathcal{K})$ by $\mathfrak{S}(\mathbb{K})$ and $\mathfrak{S}(\mathcal{K})$ respectively in the above, we get the definitions for the case of PDBL.

Theorem 13 (Soundness). For any $\alpha$ and $\beta$ in $\mathfrak{F}$,

(a) If $\alpha \vdash \beta$ is provable in DBL then it is valid in $\mathfrak{R}(\mathcal{K})$,

(b) If $\alpha \vdash \beta$ is provable in PDBL then it is valid in $\mathfrak{S}(\mathcal{K})$.

Proof. (a) As for any context $\mathbb{K} \in \mathcal{K}$ the set $\mathfrak{R}(\mathbb{K})$ of object oriented protoconcepts of $\mathbb{K}$ forms a $\mathrm{dBa}$, and for any model $\mathbb{M}:=(\mathbb{K}, v), v$ is a valuation according to Definition 12, Theorem 11 gives us the result.

(b) Replace $\mathfrak{R}(\mathbb{K})$ by $\mathfrak{S}(\mathbb{K})$ and $\mathrm{dBa}$ by pure $\mathrm{dBa}$ in the argument of (a).

Theorem 14 (Completeness). For any $\alpha$ and $\beta$ in $\mathfrak{F}$,

(a) If a sequent $\alpha \vdash \beta$ is valid in $\mathfrak{R}(\mathcal{K})$ then it is provable in $\mathbf{D B L}$,

(b) If a sequent $\alpha \vdash \beta$ is valid in $\mathfrak{S}(\mathcal{K})$ then it is provable in PDBL.

Proof. (a) If possible, suppose $\alpha \vdash \beta$ is not provable in DBL. Then by Proposition $8,[\alpha] \nsubseteq[\beta]$ in $\mathcal{L}(\mathfrak{F})$. Therefore by Proposition 1 we have either $[\alpha] \sqcap[\alpha] I_{\sqcap}$ $[\beta] \sqcap[\beta]$ or $[\alpha] \sqcup[\alpha] \nsubseteq \sqcap[\beta] \sqcup[\beta]$. Now we consider the Lindenbaum-Tarski algebra $\mathcal{L}(\mathfrak{F})$ of DBL and the context $\mathbb{K}^{c}(\mathcal{L}(\mathfrak{F})):=\left(\mathcal{F}_{p}(\mathcal{L}(\mathfrak{F})), \mathcal{I}_{p}(\mathcal{L}(\mathfrak{F})),-\Delta\right)$. By the representation Theorem 9 , there will then exist a quasi-injective homomorphism $h: \mathcal{L}(\mathfrak{F}) \rightarrow \mathfrak{R}\left(\mathbb{K}^{c}(\mathcal{L}(\mathfrak{F}))\right)$ such that $h(x):=\left(F_{\neg x}, I_{x}\right)$ for all $x \in \mathcal{L}(\mathfrak{F})$. Define the valuation $i: \mathfrak{F} \rightarrow \mathcal{L}(\mathfrak{F})$ by $i(\gamma):=[\gamma]$, for any $\gamma \in \mathfrak{F}$. Therefore composition of the two maps $v:=h \circ i$ gives a valuation from $\mathfrak{F}$ to $\mathfrak{R}\left(\mathbb{K}^{c}(\mathcal{L}(\mathfrak{F}))\right)$. So we have a model $\mathbb{M}:=\left(\mathbb{K}^{c}(\mathcal{L}(\mathfrak{F})), v\right)$.

Now if $[\alpha] \sqcap[\alpha] \Phi_{\sqcap}[\beta] \sqcap[\beta]$, there exists a prime filter $F_{0}$ in $\mathcal{L}(\mathfrak{F})_{\sqcap}$ (a Boolean algebra, by Proposition 1$)$ such that $[\alpha] \sqcap[\alpha] \in F_{0}$ and $[\beta] \sqcap[\beta] \notin F_{0}$. Therefore by Lemma 1 there exists a filter $F$ in $\mathcal{L}(\mathfrak{F})$ such that $F \cap \mathcal{L}(\mathfrak{F})_{\sqcap}=F_{0}$ and as $F_{0}$ is prime, $F \in \mathcal{F}_{p}(\mathcal{L}(\mathfrak{F}))$. As $[\alpha] \sqcap[\alpha] \in F_{0},[\alpha] \sqcap[\alpha] \in F$ and $[\beta] \sqcap[\beta] \notin$ $F$ as $[\beta] \sqcap[\beta] \notin F_{0}$. So $[\alpha] \in F$ as $[\alpha] \sqcap[\alpha] \sqsubseteq[\alpha]$, and $[\beta] \notin F$ otherwise $[\beta] \sqcap[\beta] \in F$. This gives $F \notin F_{\neg[\alpha]}$ and $F \in F_{\neg[\beta]}$, whence $F_{\neg[\beta]} \nsubseteq F_{\neg[\alpha]}$. So $v(\alpha)=\left(F_{\neg[\alpha]}, I_{[\alpha]}\right) \nsubseteq\left(F_{\neg[\beta]}, I_{[\beta]}\right)=v(\beta)$ by Proposition 4 . 
In case $[\alpha] \sqcup[\alpha] \nsubseteq \sqcap[\beta] \sqcup[\beta]$, we can dually show that there exists $I \in \mathcal{I}_{p}(\mathcal{L}(\mathfrak{F}))$ such that $[\alpha] \notin I$ and $[\beta] \in I$ giving $I_{[\beta]} \nsubseteq I_{[\alpha]}$.

So $\alpha \vdash \beta$ is not true in $\mathfrak{R}\left(\mathbb{K}^{c}(\mathcal{L}(\mathfrak{F}))\right)$, which is not possible as $\alpha \vdash \beta$ is valid in $\mathfrak{R}(\mathcal{K})$. Hence we get a contradiction.

A similar argument using the result for PDBL corresponding to Proposition 8 and the representation Theorem 6 , gives (b).

\section{Conclusion}

This work proposes the notion of object oriented protoconcepts, and characterizes them in terms of approximations by object oriented concepts. A representation result is obtained, showing that any double Boolean algebra is quasiembeddable in an algebra of object oriented protoconcepts. A logic DBL for the class of object oriented protoconcepts is defined and extended to a logic PDBL for the class of pure double Boolean algebras. Using the representation results for double and pure double Boolean algebras, the logics are shown to be sound and complete with respect to the class of object oriented protoconcepts and semiconcepts over the class of all contexts respectively. As further work, one can investigate representation results for the algebras that yield isomorphisms. On the side of the logics, other semantics could be explored - for instance, a Kripke-style semantics that may be in the line of work done in [2].

\section{References}

1. Balbiani, P.: Deciding the word problem in pure double Boolean algebras. J. Appl. Logic 10, 260-273 (2012)

2. Conradie, W., Frittella, S., Palmigiano, A., Piazzai, M., Tzimoulis, A., Wijnberg, N.M.: Toward an epistemic-logical theory of categorization. In: Electronic Proceedings in Theoretical Computer Science, EPTCS, TARK 2017, vol. 251, pp. 167-186 (2017)

3. Davey, B.A., Priestley, H.A.: Introduction to Lattices and Order. Cambridge University Press, Cambridge (2002)

4. Düntsch, I., Gediga, G.: Modal-style operators in qualitative data analysis. In: Proceedings of 2002 IEEE International Conference on Data Mining, pp. 155-162. IEEE (2002)

5. Ganter, B., Wille, R.: Formal Concept Analysis: Mathematical Foundations. Springer, Heidelberg (2012)

6. Howlader, P., Banerjee, M.: Algebras from semiconcepts in rough set theory. In: Nguyen, H.S., Ha, Q.-T., Li, T., Przybyła-Kasperek, M. (eds.) IJCRS 2018. LNCS (LNAI), vol. 11103, pp. 440-454. Springer, Cham (2018). https://doi.org/10.1007/ 978-3-319-99368-3_34

7. Kwuida, L.: Prime ideal theorem for double Boolean algebras. Discuss. Math.General Algebra Appl. 27(2), 263-275 (2007)

8. Vormbrock, B.: A solution of the word problem for free double boolean algebras. In: Kuznetsov, S.O., Schmidt, S. (eds.) ICFCA 2007. LNCS (LNAI), vol. 4390, pp. 240-270. Springer, Heidelberg (2007). https://doi.org/10.1007/978-3-540-709015_16 
9. Vormbrock, B., Wille, R.: Semiconcept and protoconcept algebras: the basic theorems. In: Ganter, B., Stumme, G., Wille, R. (eds.) Formal Concept Analysis. LNCS (LNAI), vol. 3626, pp. 34-48. Springer, Heidelberg (2005). https://doi.org/ $10.1007 / 11528784 \_2$

10. Wille, R.: Concept lattices and conceptual knowledge systems. Comput. Math. Appl. 23(6-9), 493-515 (1992)

11. Wille, R.: Boolean concept logic. In: Ganter, B., Mineau, G.W. (eds.) ICCSConceptStruct 2000. LNCS (LNAI), vol. 1867, pp. 317-331. Springer, Heidelberg (2000). https://doi.org/10.1007/10722280_22

12. Yang, L., Luoshan, X.: On rough concept lattices. Electron. Notes Theor. Comput. Sci. 257, 117-133 (2009)

13. Yao, Y.: A comparative study of formal concept analysis and rough set theory in data analysis. In: Tsumoto, S., Słowiński, R., Komorowski, J., Grzymała-Busse, J.W. (eds.) RSCTC 2004. LNCS (LNAI), vol. 3066, pp. 59-68. Springer, Heidelberg (2004). https://doi.org/10.1007/978-3-540-25929-9_6

14. Yao, Y.: Concept lattices in rough set theory. In: 2004 Annual Meeting of the North American Fuzzy Information Processing Society, vol. 2, pp. 796-801. IEEE (2004)

15. Yao, Y., Chen, Y.: Rough set approximations in formal concept analysis. In: Peters, J.F., Skowron, A. (eds.) Transactions on Rough Sets V. LNCS, vol. 4100, pp. 285305. Springer, Heidelberg (2006). https://doi.org/10.1007/11847465_14 